\title{
Comparative Analysis of Impurity Transport in the Peripheral Plasma in the Large Helical Device for Carbon and Tungsten Divertor Configurations with EMC3-EIRENE*)
}

\author{
Mamoru SHOJI $^{1)}$ and Gakushi KAWAMURA ${ }^{1,2)}$ \\ ${ }^{1)}$ National Institute for Fusion Science, National Institutes of Natural Sciences, Toki 509-5292, Japan \\ ${ }^{2)}$ Department of Fusion Science, Graduate University for Advanced Studies (SOKENDAI), Toki 509-5292, Japan
}

(Received 27 December 2018 / Accepted 17 February 2019)

\begin{abstract}
Impurity transport in the peripheral plasma in the Large Helical Device for carbon and tungsten divertor configurations is analyzed using a three-dimensional edge plasma simulation code (EMC3-EIRENE). Long pulse plasma discharges in LHD have often been interrupted by the emission of iron dust from the surface of the vacuum vessel. The iron ions in the peripheral plasma originating from the iron dust can enhance the sputtering of impurities on the divertor plates. The influence on the peripheral plasma by the sputtered impurities is investigated in the iron dust emission case for both the carbon and the tungsten divertor configurations. The simulation reveals that the dependence of the radiation power by the sputtered impurities on the plasma density and the iron emission rate is quite different between the two divertor configurations. It is found that, for the tungsten divertor, a high plasma density operation is advantageous for controlling the radiation power and the impurity ion content. In the high plasma density operation, the radiation power by tungsten ions is suppressed to less than that by carbon ions for the carbon divertor even in the case of high iron dust emission rates due to the reduced sputtering rate by lowered divertor electron temperatures.
\end{abstract}

(C) 2019 The Japan Society of Plasma Science and Nuclear Fusion Research

Keywords: impurity transport, tungsten, carbon, divertor, EMC3-EIRENE, LHD

DOI: $10.1585 /$ pfr.14.3403057

\section{Introduction}

Recent long pulse discharges in the Large Helical Device (LHD) have often been interrupted by a carbon dust emission from the divertor region caused by the exfoliation of carbon dominant mixed-material deposition layers $[1,2]$. The conventional carbon divertor plates will be replaced to tungsten plates in near future for suppressing the deposition layers. It is expected that the newly installed tungsten divertor plates will contribute to the extension of the duration time of the long pulse discharges as a result of the low sputtering rate of tungsten. However, the sputtered tungsten can interrupt the plasma discharges by the higher cooling rate due to highly charged tungsten ions in the plasma. For adopting the tungsten divertor, there is a concern about the sustainment of long pulse discharges. That is, electric arcing on the surface of the vacuum vessel has been frequently observed during the plasma discharges with abrupt increase in the iron ion intensity in the plasma, where large amounts of iron dust were released by the electric arcing [3]. The dust enters the peripheral plasma, and iron ions are produced because iron is the main component of the vacuum vessel (SUS316L). The iron ions can trigger

author'se-mail: shoji@nifs.ac.jp

*) This article is based on the presentation at the 27th International Toki Conference (ITC27) \& the 13th Asia Pacific Plasma Theory Conference (APPTC2018) the physical sputtering of tungsten on the divertor plates by acceleration of the iron ions by the sheath potential formed in front of the divertor plates.

In order to understand the characteristics of the tungsten divertor compared to that of the conventional carbon divertor, a fully three-dimensional edge plasma simulation code (EMC3-EIRENE) is applied [4,5]. The influence on the peripheral plasma by the sputtered impurities (carbon and tungsten) is surveyed in various plasma densities, plasma heating powers, and iron emission rates for both the divertor configurations. The simulations contribute to not only finding the optimized experimental condition for suppressing the influence of the sputtered impurities, but also to understanding the characteristics of both the carbon and the tungsten divertor configurations in more detail.

\section{Setup of Impurity Transport Simu- lation in the Peripheral Plasma}

The EMC3-EIRENE calculates a steady-state solution of the three-dimensional profile of peripheral plasma parameters including the effect of the impurity ion transport. The simulation is performed in a three-dimensional grid model as shown in Fig. 1. This model includes an ergodic layer around the Last Closed Flux Surface (LCFS), divertor legs, divertor components, and the vacuum vessel for 


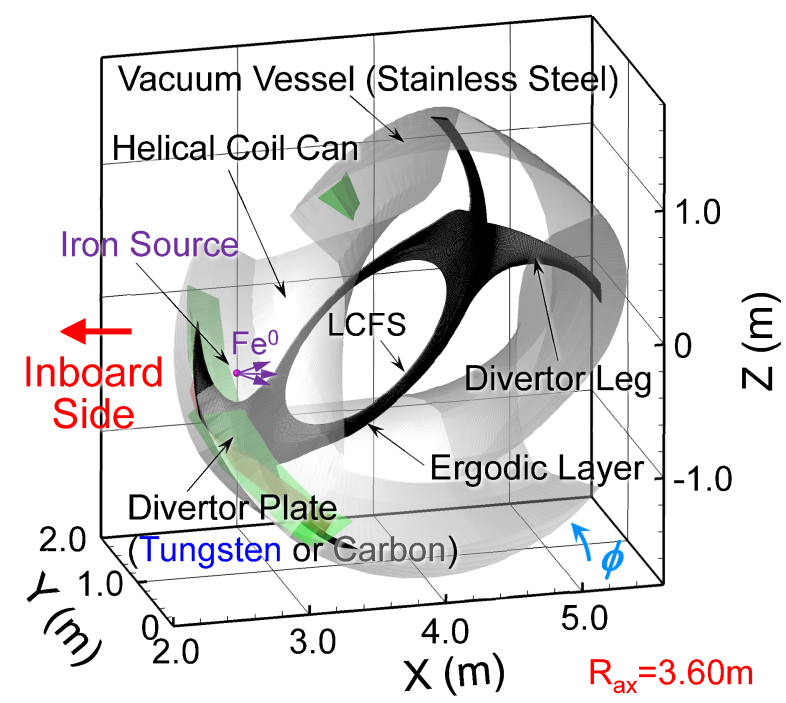

Fig. 1 A perspective view of a three-dimensional grid model used in the EMC3-EIRENE for a typical magnetic configuration $\left(R_{\mathrm{ax}}=3.60 \mathrm{~m}\right)$ in the case of the iron dust emission. The poloidal cross-section of the grid cells for the LHD peripheral plasma at the toroidal angle where the iron source exists $\left(\phi=10.625^{\circ}\right)$ is described as a group of small fine black cells.

one-half of the helical coil pitch angle $\left(0^{\circ} \leq \phi \leq 18^{\circ}\right.$ in toroidal direction $\phi$ ), in which the radial position of the magnetic axis $R_{\mathrm{ax}}$ is set to be $3.60 \mathrm{~m}$ (one of the most typical magnetic configurations in LHD). At both toroidal edges of the model, a periodical boundary condition is adopted. The material on the surface of the vacuum vessel is regarded as iron. All the divertor components consist of tungsten/carbon for tungsten/carbon divertor configurations, respectively.

The impurity ion density profile in the peripheral plasma is obtained by solving the particle and the momentum balance equations of the impurity ions along magnetic field lines under the assumption that the impurity ion temperature is equal to the plasma ion temperature. The radiation power by the impurities and the ionization/recombination coefficients are derived from the database on the Atomic Data and Analysis Structure (ADAS) [6]. The position of the iron source locates on a surface on a helical coil can in the inboard side of the torus. This position corresponds to the location where electric arcing was actually initiated at the termination of a long pulse discharge [3]. A number of test particles representing iron atoms (two million at the maximum) with a kinetic energy corresponding to the boiling temperature of iron $(0.26 \mathrm{eV})$ are launched from this position to deuterium plasmas.

The perpendicular particle and thermal diffusion coefficients $\left(D_{\perp}\right.$ and $\left.\chi_{\perp}\right)$ are assumed to be $0.5 \mathrm{~m}^{2} / \mathrm{s}$ and $1.5 \mathrm{~m}^{2} / \mathrm{s}$, respectively. The profile of the plasma parameters and the impurities is obtained as a converged solution. It is found that the EMC3-EIRENE cannot provide the converged solution when the averaged divertor electron temperature is less than several $\mathrm{eV}$. Three input parameters must be defined for carrying out the simulation. The first and second ones are the plasma heating power $P^{\mathrm{LCFS}}$ and the plasma density $n_{\mathrm{e}}^{\mathrm{LCFS}}$ at the core-SOL boundary (defined just inside the LCFS). The third parameter is the emission rate of the iron atoms $I^{\mathrm{Fe} 0}$ released from the position of the iron source. The bulk neutral particles (deuterium atoms and molecules) are recycled in the grid model (no bulk particle sink exists). The impurity ions arriving at the core-SOL boundary are reflected to the peripheral plasma while maintaining the charge states of the ions. The impurity atoms/ions reaching to the divertor components or the vacuum vessel are treated along the following procedures.

The original EMC3-EIRENE does not include physical sputtering (chemical and self-sputtering have already been implemented in the code). Thus, a variable chemical sputtering rate has been newly introduced as a pseudosputtering rate which includes both the chemical and the physical sputtering rates $[7,8]$. The value of this sputtering rate is changed during the iterative calculation processes for obtaining the converged solution. The sputtering rate is varied with the averaged electron and ion temperature in front of the divertor plates taking account of the charge state of the incident impurity ions [9]. An energy of $10 \mathrm{eV}$ is adopted as the kinetic energy of the sputtered tungsten atoms from the tungsten divertor plates by both the physical $(\mathrm{Fe} \rightarrow \mathrm{W})$ and the self-sputtering $(\mathrm{W} \rightarrow \mathrm{W})$ [10]. An energy of $0.05 \mathrm{eV}$ is set for the kinetic energy of the sputtered carbon atoms from the carbon divertor plates, which equals the energy of the typical divertor plate temperature in plasma discharges. The kinetic energy of the carbon atoms sputtered by the self-sputtering process $(\mathrm{C} \rightarrow \mathrm{C})$ is set to be $20 \mathrm{eV}$ [7]. The reflection of the incident iron atoms and the tungsten/carbon atoms are not included. The influence of this assumption on the simulation results is estimated to be less than a few percent at most because of the much lower reflection coefficients than the sputtering yields of the impurities.

\section{Impurity Transport Analysis \\ 3.1 Simulation results for the carbon diver- tor}

Figure 2 shows the poloidal cross-section of the calculated profile of the carbon and iron ion densities, the radiation power by total carbon ions (including carbon ions with all charge states), and the electron temperature in the peripheral plasma for the carbon divertor. The emission rate of the iron atoms $I^{\mathrm{Fe} 0}$ is set to be $6.24 \times 10^{19}$ atoms/s. The plasma heating power and the density at the core-SOL boundary are set to be $12 \mathrm{MW}$ and $1 \times 10^{19} \mathrm{~m}^{-3}$ (a low plasma density condition), respectively. This figure shows that while the singly charged carbon ions $\left(\mathrm{C}^{1+}\right)$ is localized in both the divertor legs and the outer edge of the ergodic 
Carbon Divertor, $\mathrm{PLCFS}=12 \mathrm{MW}, \underline{\mathrm{n}_{\underline{e}}} \underline{\mathrm{LCFS}}=1 \times 10^{19} \mathrm{~m}^{-3}, \mathrm{IFe}=6.24 \times 10^{19}$ atoms $/ \mathrm{s}$
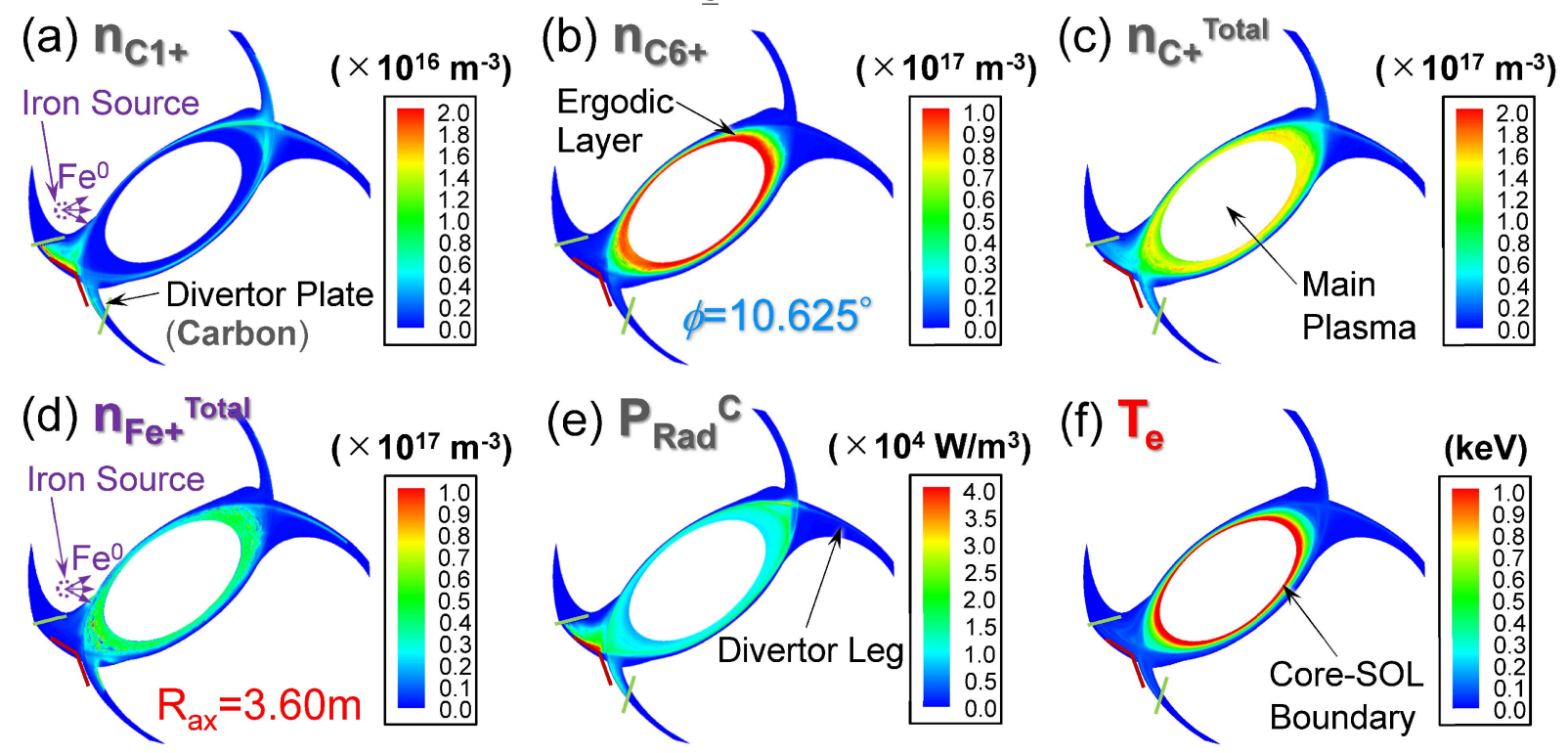

Fig. 2 The poloidal cross-section of the profile of the singly charged carbon ions (a), highly charged carbon ions (b), the total carbon ion density (c), the total iron ion density (d), the radiation power by the carbon ions (e), and the electron temperature in the peripheral plasma (f) for the carbon divertor configuration in a low plasma density condition $\left(P^{\mathrm{LCFS}}=12 \mathrm{MW}, n_{\mathrm{e}}^{\mathrm{LCFS}}=1 \times 10^{19} \mathrm{~m}^{-3}\right.$, and $I^{\mathrm{Fe} 0}$ $=6.24 \times 10^{19}$ atoms $\left./ \mathrm{s}\right)$.

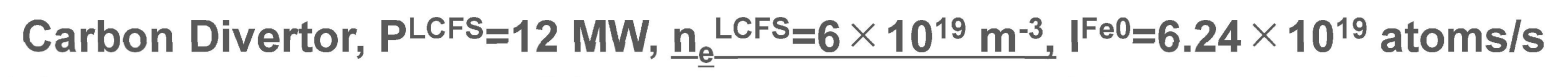
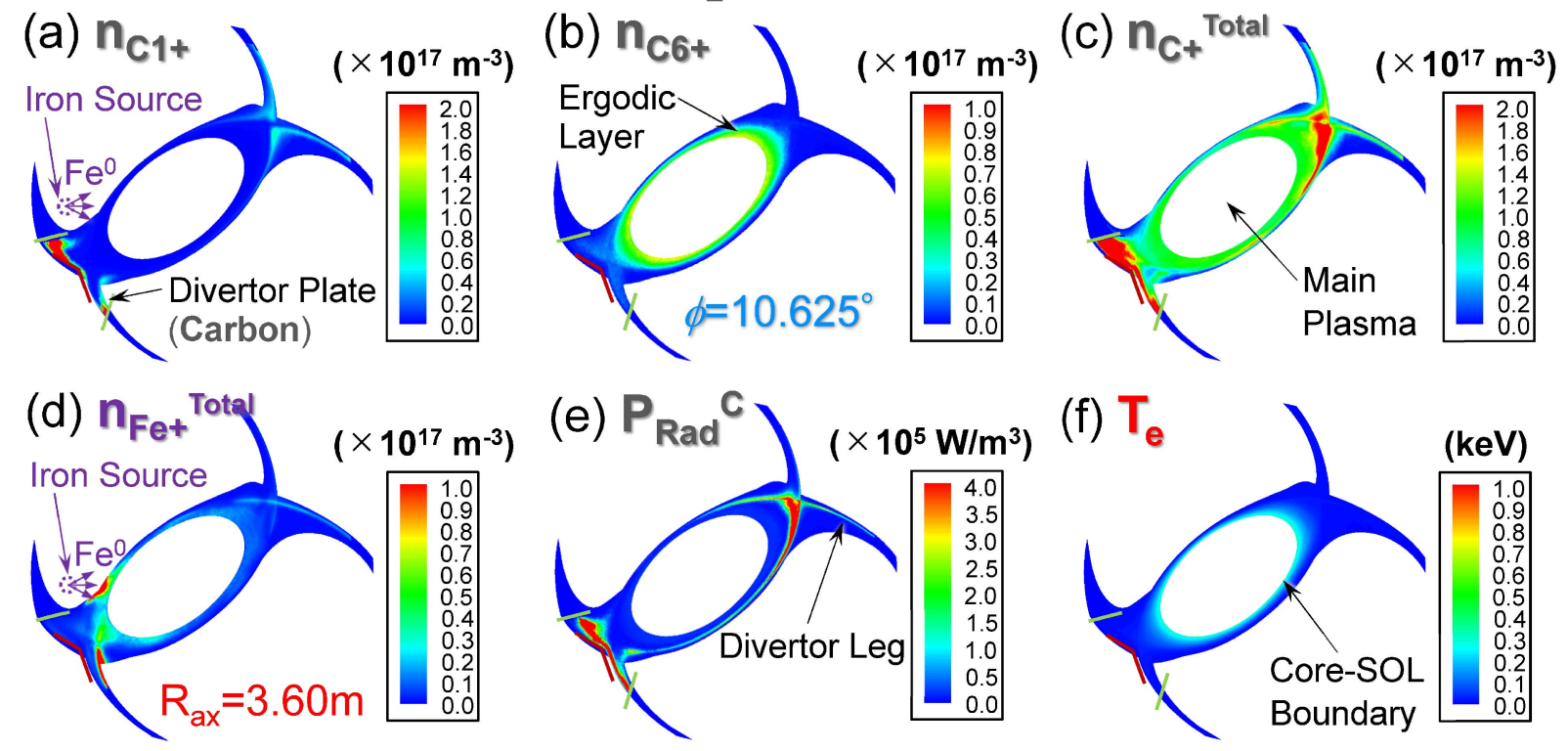

Fig. 3 The poloidal cross-section of the profile of the singly charged carbon ions (a), highly charged carbon ions (b), the total carbon ion density (c), the total iron ion density (d), the radiation power by the carbon ions (e), and the electron temperature in the peripheral plasma (f) for the carbon divertor configuration in a high plasma density condition ( $P^{\mathrm{LCFS}}=12 \mathrm{MW}, n_{\mathrm{e}}^{\mathrm{LCFS}}=6 \times 10^{19} \mathrm{~m}^{-3}$, and $I^{\mathrm{Fe} 0}=6.24 \times 10^{19}$ atoms $\left./ \mathrm{s}\right)$.

layer (Fig. 2 (a)), the highly charged carbon ions $\left(\mathrm{C}^{6+}\right)$ are distributed around the core-SOL boundary (Fig. 2 (b)). The density of the total iron ions is also distributed around the core-SOL boundary (Fig. 2 (d)).

Figure 3 presents the poloidal cross-section of the calculated parameters for a high plasma density condition where $P^{\mathrm{LCFS}}$ and $n_{\mathrm{e}}^{\mathrm{LCFS}}$ are $12 \mathrm{MW}$ and $6 \times 10^{19} \mathrm{~m}^{-3}$, respectively. Being different from the simulation for the low plasma density condition, the total iron ion density is locally high near the iron source (Fig. $3(\mathrm{~d})$ ). The radiation power by the total carbon ions is observable in both the outer edge of the ergodic layer and the divertor legs as 


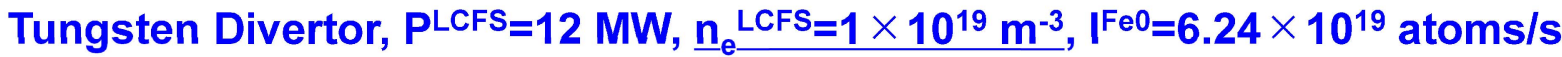
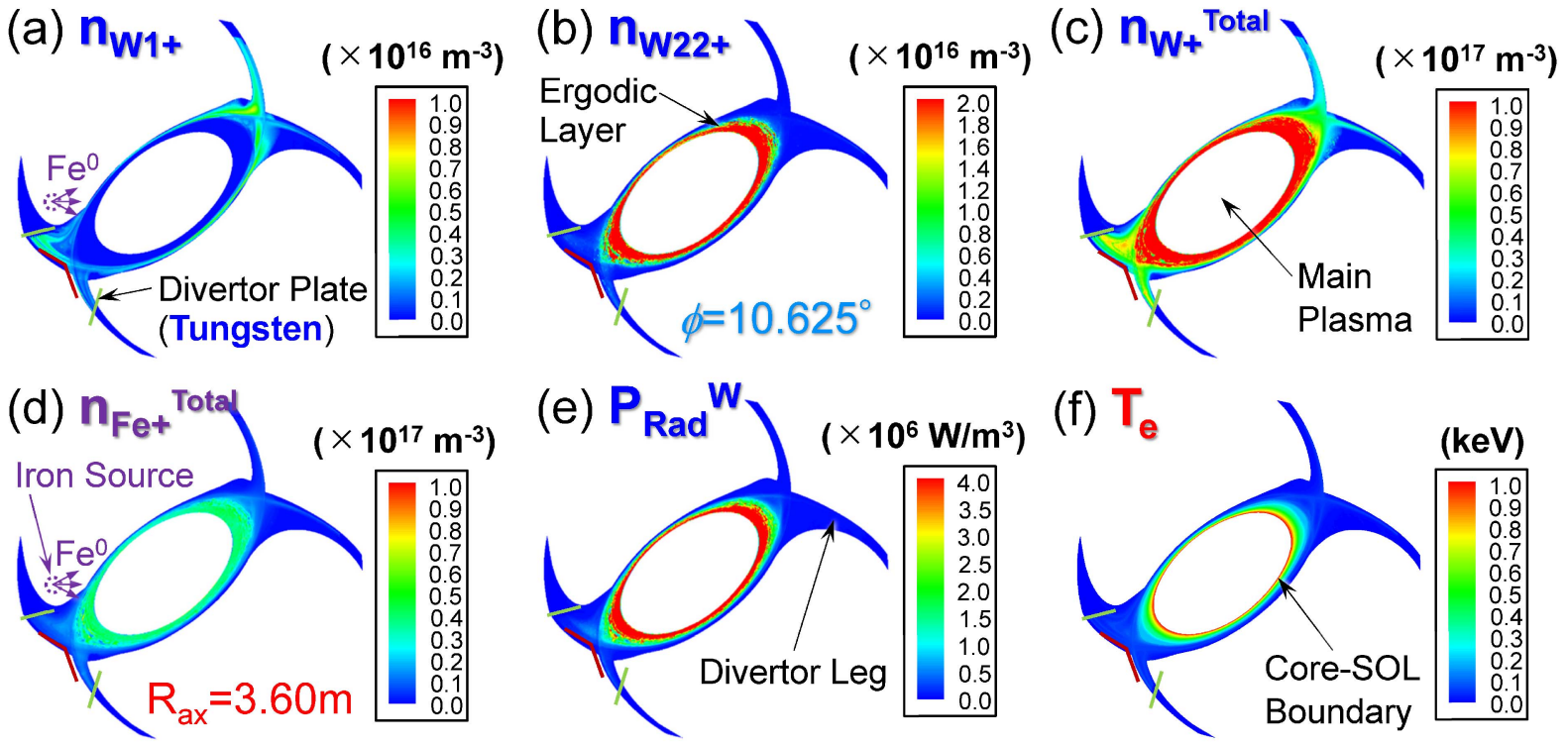

Fig. 4 The poloidal cross-section of the profile of the singly charged tungsten ions (a), moderately charged tungsten ions (b), the total tungsten ion density (c), the total iron ion density (d), the radiation power by the tungsten ions (e), and the electron temperature in the peripheral plasma for the tungsten divertor configuration in a low plasma density condition $\left(P^{\mathrm{LCFS}}=12 \mathrm{MW}, n_{\mathrm{e}}^{\mathrm{LCFS}}=1 \times\right.$ $10^{19} \mathrm{~m}^{-3}$, and $I^{\mathrm{Fe} 0}=6.24 \times 10^{19}$ atoms $/ \mathrm{s}$ ).

shown in Fig. 3 (e). It has been recognized that the significant difference of both the iron/carbon ion density and the radiation power by the carbon ions for the low and high plasma densities is explained by the balance between the thermal force and the friction force in the two plasma density conditions [11].

\subsection{Simulation results for the tungsten diver- tor}

Figure 4 illustrates the poloidal cross-section of the calculated profiles in the low plasma density condition for the tungsten divertor. While the singly charged tungsten ions $\left(\mathrm{W}^{1+}\right)$ exist in the outer edge of the ergodic layer and in the divertor legs (Fig. 4(a)), the moderately charged tungsten ions $\left(\mathrm{W}^{22+}\right)$ are distributed around the core-SOL boundary (Fig. 4(b)), which is the same as those for the carbon divertor in the low plasma density condition (Figs. 2 (a) and (b)).

Figure 5 gives the poloidal cross-section of the calculated profiles in the high plasma density for the tungsten divertor. The density of the singly charged tungsten ions $\left(\mathrm{W}^{1+}\right)$ is quite low and it is localized near the tungsten divertor plates (Fig. 5 (a)). Compared to the simulations for the low plasma density, the total tungsten ion density (Fig. 5 (c)) and the radiation power (Fig. 5 (e)) are significantly suppressed. The total iron density is concentrated near the iron source (Fig. 5 (d)). This considerable difference for the low and high plasma densities is also explained by the balance between the two forces in the two different plasma density conditions [11].

\section{The Dependence of the Impurity Transport on the Plasma Density}

\subsection{Simulation results for the carbon diver- tor}

The impurity transport simulation was performed in various plasma densities $n_{\mathrm{e}}^{\mathrm{LCFS}}$ and plasma heating powers $P^{\mathrm{LCFS}}$ for the carbon divertor. The ranges of the both two parameters are between $1 \times 10^{19} \mathrm{~m}^{-3}$ and $7 \times 10^{19} \mathrm{~m}^{-3}$, and between $1 \sim 2 \mathrm{MW}$ and $12 \mathrm{MW}$, respectively. These parameter ranges thoroughly cover the most of typical operational conditions in LHD plasma discharge experiments [12]. Figure 6 gives the simulation results which show the radiation power by the carbon ions $\left(P_{\mathrm{Rad}}^{\mathrm{C}}\right)$, the total carbon ion content in the peripheral plasma, the averaged divertor electron temperature, the production rate of the singly charged carbon ions, the radiation power by the iron ions, and the total iron ion content, respectively. The radiation power and the impurity ion content are calculated by summing those with all charge states in all grid cells.

While the divertor electron temperature decreases with the plasma density (Fig. 6(c)), the radiation power by the carbon ions (Fig. 6(a)) and the carbon ion production rate (Fig. 6 (c)) gradually increase with the plasma density, which is explained by the almost constant sputtering rate of carbon which is irrelevant to the divertor plasma temperature. In other words, the sputtered carbon flux linearly increases with the deuterium ion flux onto the divertor plates. The radiation power by the iron ions (Fig. 6 (e)) and the iron ion content (Fig. 6 (f)) do not significantly change with the plasma density and the plasma heating power because 
Tungsten Divertor, PLCFS $=12 \mathrm{MW}, \underline{\mathrm{n}_{\mathrm{e}}} \underline{\mathrm{LCFS}}=6 \times 10^{19} \mathrm{~m}^{-3}, \mathrm{IFe}=6.24 \times 10^{19}$ atoms $/ \mathrm{s}$
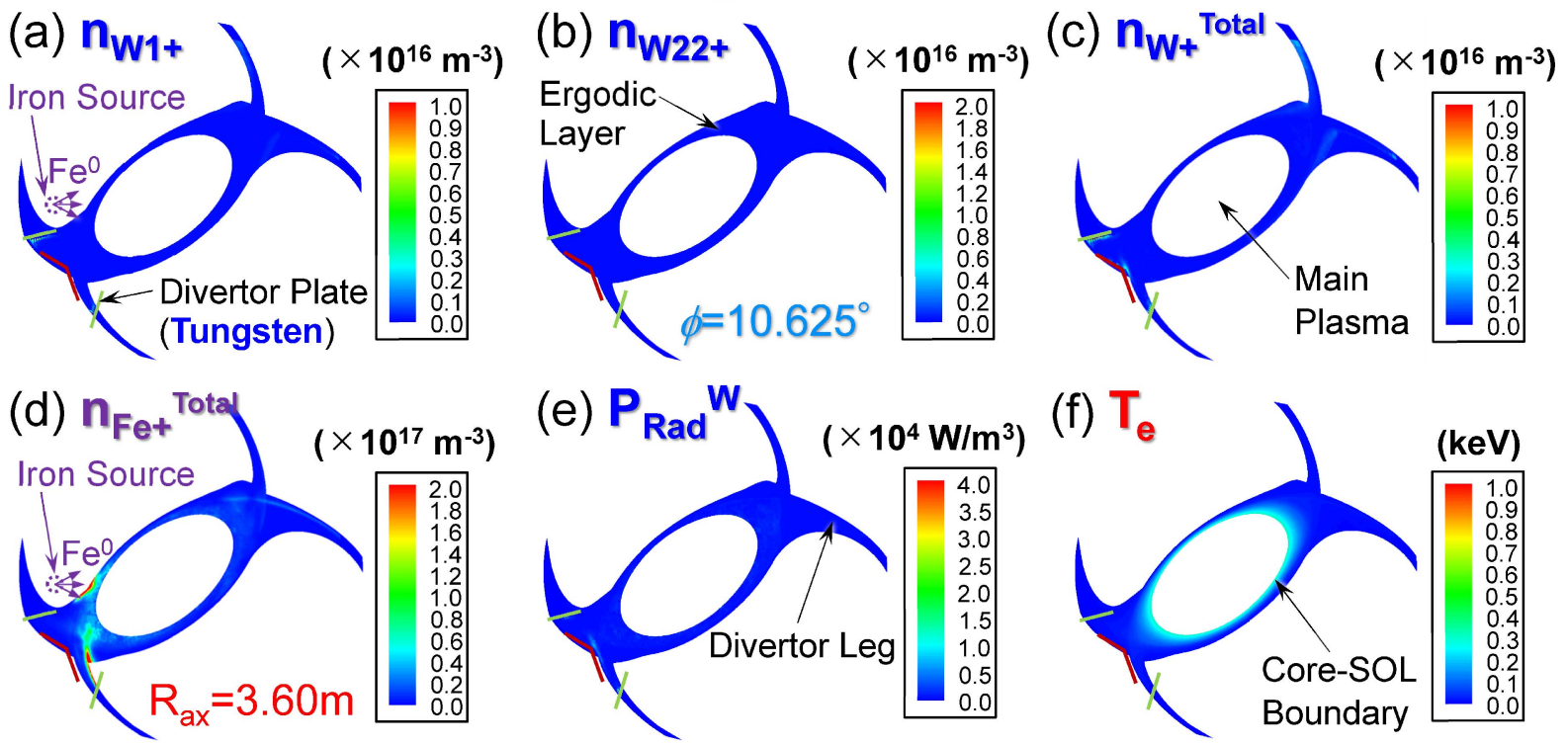

Fig. 5 The poloidal cross-section of the profile of the singly charged tungsten ions (a), moderately charged tungsten ions (b), the total tungsten ion density (c), the total iron ion density (d), the radiation power by the tungsten ions (e), and the electron temperature in the peripheral plasma for the tungsten divertor configuration in a high plasma density condition $\left(P^{\mathrm{LCFS}}=12 \mathrm{MW}, n_{\mathrm{e}}^{\mathrm{LCFS}}=6 \times\right.$ $10^{19} \mathrm{~m}^{-3}$, and $I^{\mathrm{Fe} 0}=6.24 \times 10^{19}$ atoms $\left./ \mathrm{s}\right)$.
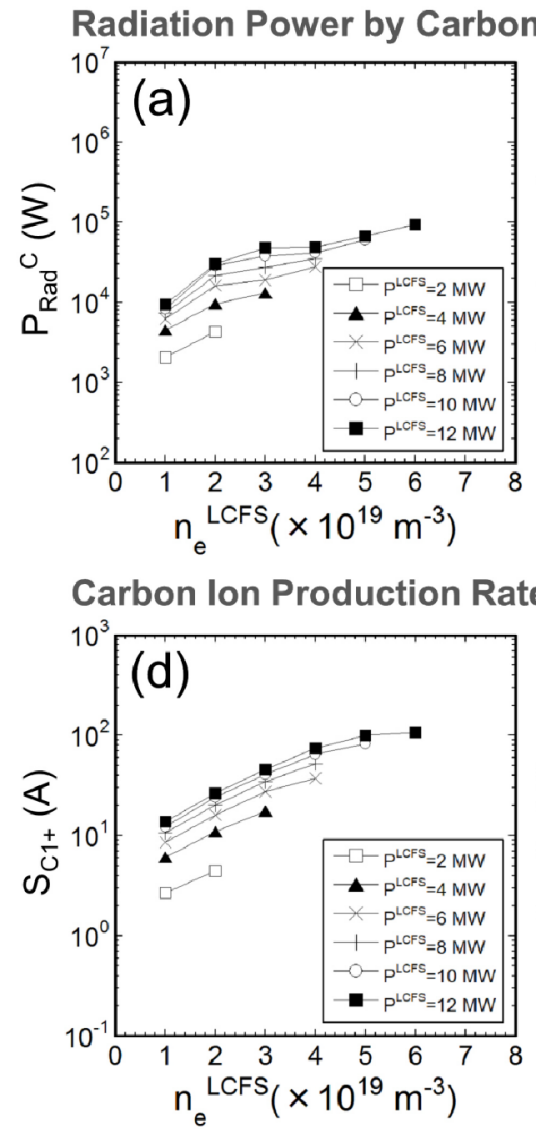
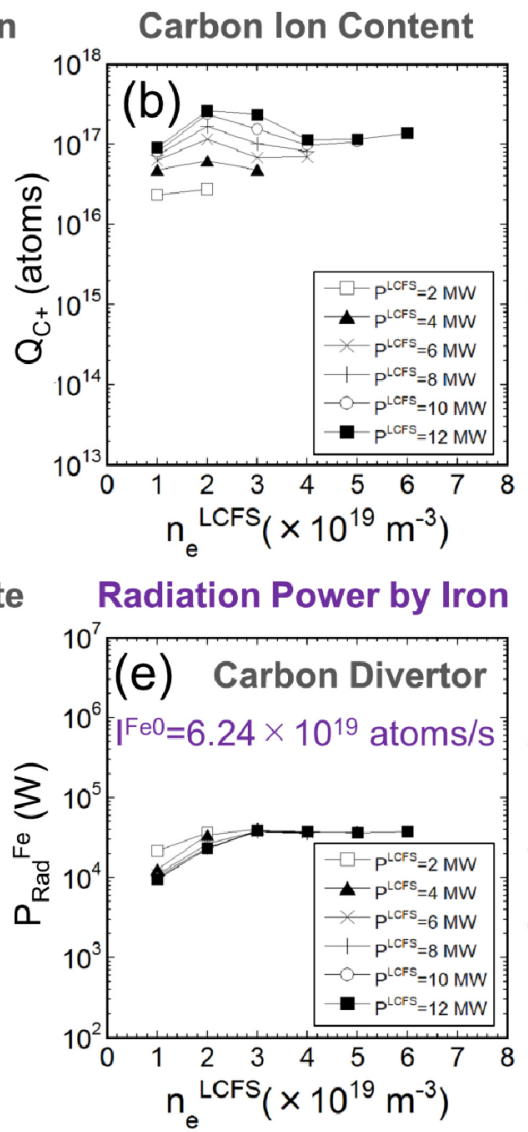
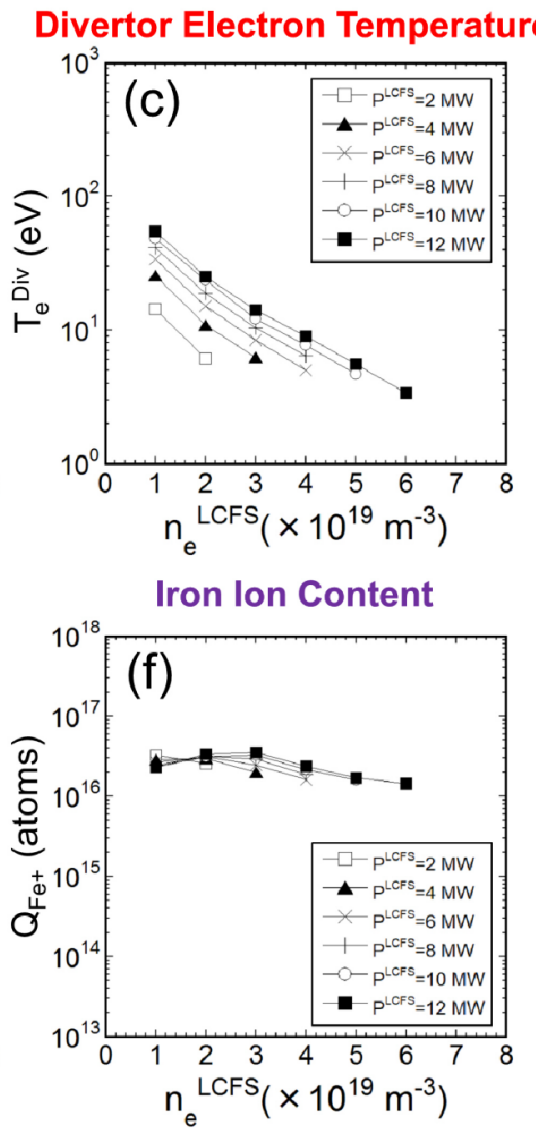

Fig. 6 The simulation results showing the dependence of the radiation power by the carbon ions (a), the total carbon ion content (b), the divertor electron temperature (c), the production rate of the singly charged carbon ions (d), the radiation power by the iron ions (e), and the total iron ion content (f) in the peripheral plasma on the plasma density $n_{\mathrm{e}}^{\mathrm{LCFS}}$ and the heating power $P^{\mathrm{LCFS}}$ for the carbon divertor. 

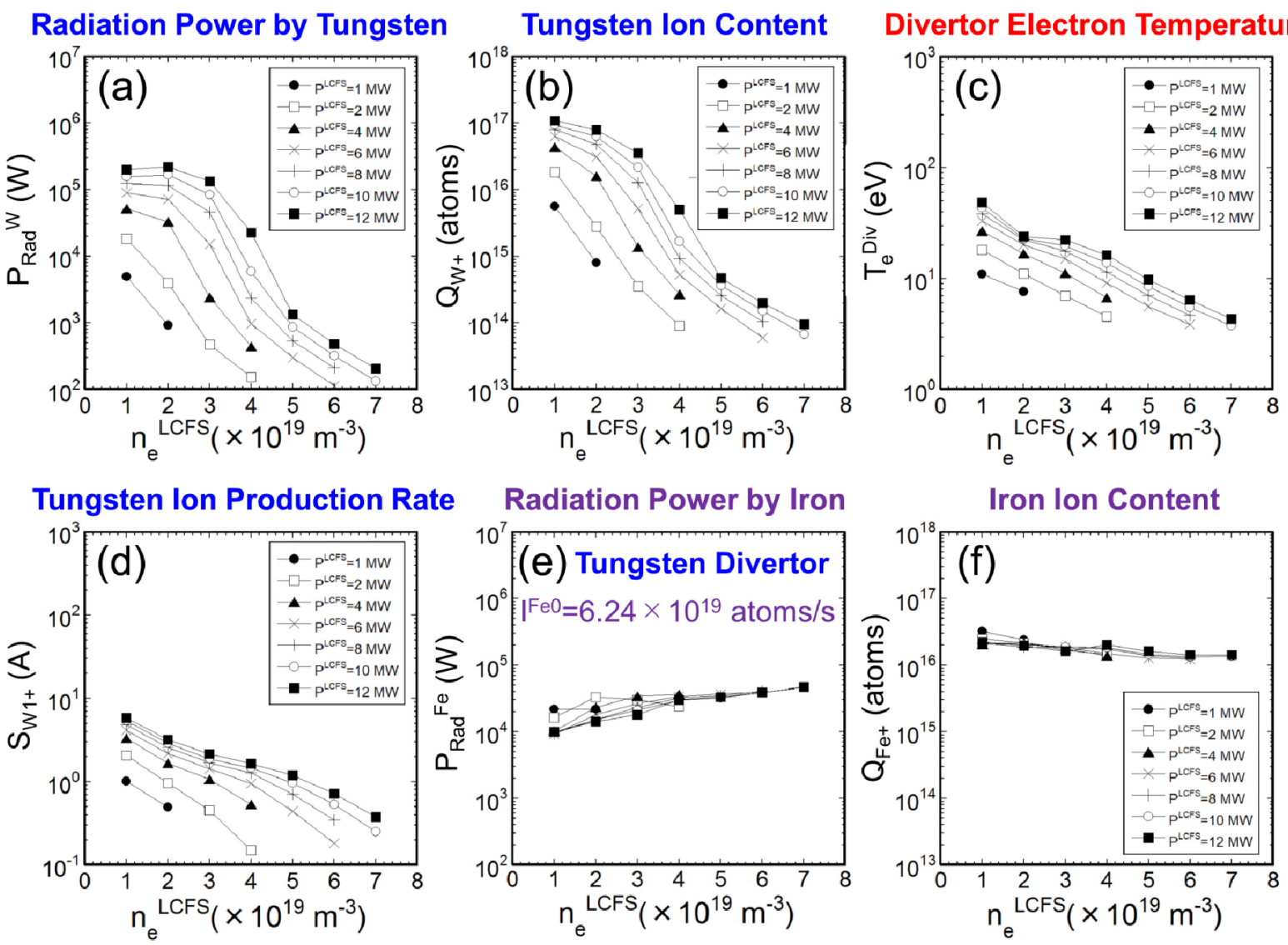

Fig. 7 The dependence of the radiation power by the tungsten ions (a), the total tungsten ion content (b), the divertor electron temperature (c), the production rate of the singly charged tungsten ions (d), the radiation power by the iron ions (e), and the total iron ion content (f) in the peripheral plasma on the plasma density $n_{e}^{\mathrm{LCFS}}$ and the heating power $P^{\mathrm{LCFS}}$ for the tungsten divertor.

of the constant emission rate of the iron atoms in the simulation.

\subsection{Simulation results for the tungsten diver- tor}

For the tungsten divertor, the radiation power by the tungsten ions (Fig. 7 (a)), the total tungsten ion content (Fig. 7 (b)) in the peripheral plasma, and the singly charged tungsten ion production rate (Fig. 7 (d)) drastically decrease with the plasma density. These simulation results are completely different from those for the carbon divertor (Figs. 6(a), (b), and (d)). The simulation has revealed that this trend for high plasma densities $\left(n_{\mathrm{e}}^{\mathrm{LCFS}} \geq 4 \times 10^{19} \mathrm{~m}^{-3}\right)$ is explained by the synergy effect of both the reduction of the physical/self-sputtering rate due to the lowered divertor electron temperature and the dominant friction force on the tungsten ions in the peripheral plasma [9]. The simulation results indicate that, for the tungsten divertor, a higher plasma density operation is effective for suppressing the tungsten ions induced by the iron dust emission. For example, in the low plasma density and high plasma heating power condition $\left(n_{\mathrm{e}}^{\mathrm{LCFS}}=1 \times 10^{19} \mathrm{~m}^{-3}\right.$ and $P^{\mathrm{LCFS}}=$ $12 \mathrm{MW})$, the simulation predicts that the radiation power by tungsten ions $\left(P_{\mathrm{Rad}}^{\mathrm{W}}\right)$ in the tungsten divertor configuration is larger than that by carbon ions $\left(P_{\text {Rad }}^{\mathrm{C}}\right)$ in the carbon divertor configuration by about 20 . On the other hand, in the high plasma density condition $\left(n_{\mathrm{e}}^{\mathrm{LCFS}}=7 \times 10^{19} \mathrm{~m}^{-3}\right.$ and $P^{\mathrm{LCFS}}=12 \mathrm{MW}$ ), the radiation power by tungsten ions $\left(P_{\mathrm{Rad}}^{\mathrm{W}}\right)$ is significantly reduced to lower than that by carbon ions $\left(P_{\mathrm{Rad}}^{\mathrm{C}}\right)$ by about three-orders of magnitude as shown in Fig. 6 (a) and Fig. 7 (a).

\section{The Dependence of the Impurity Transport on the Iron Emission Rate}

\subsection{Simulation results for the carbon diver- tor}

The impurity transport in the LHD peripheral plasma is investigated in various iron emission rates for the carbon divertor in the case of a plasma heating power $P^{\mathrm{LCFS}}$ of $12 \mathrm{MW}$. Figure 8 presents the dependence of the radiation power by the carbon ions, the total carbon ion content, the divertor electron temperature, the production rate of the singly charged carbon ions, the radiation power by the iron ions, and the total iron ion content in various plasma density conditions. The radiation power by the carbon ions (Fig. 8 (a)) and the total carbon ion content (Fig. 8 (b)) do not significantly change with the iron emission rate. The production rate of the singly charged 

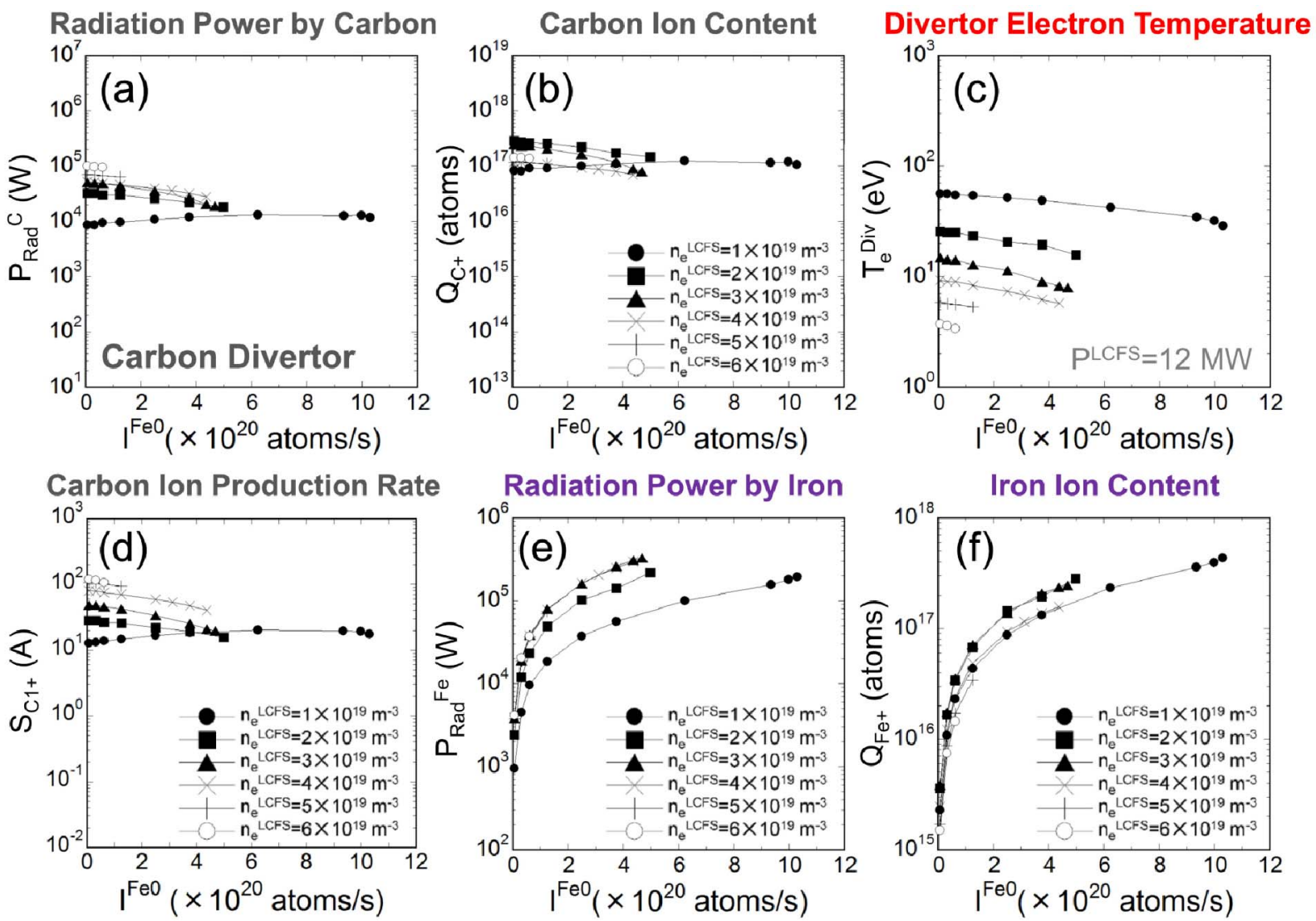

Fig. 8 The dependence of the radiation power by the carbon ions (a), the total carbon ion content (b), the divertor electron temperature (c), the production rate of the singly charged carbon ions (d), the radiation power by the iron ions (e), and the total iron ion content (f) in the peripheral plasma on the iron emission rate $I^{\mathrm{Fe} 0}$ and the plasma heating power $P^{\mathrm{LCFS}}$ for the carbon divertor in the case of $P^{\mathrm{LCFS}}=12 \mathrm{MW}$.

carbon ions (Fig. 8 (d)) shows a similar trend to that of the radiation power and the carbon ion content. The radiation power by the iron ions (Fig. 8(e)) and the total iron ion content (Fig. 8 (f)) increase with the iron emission rate. The divertor electron temperature slightly decreases with the iron emission rate (Fig. 8 (c)).

Figure 9 depicts the poloidal cross-section of the profile of the radiation power by the total iron ions, by the total carbon ions, and the electron temperature profile in the carbon divertor for low and high iron emission rates $\left(I^{\mathrm{Fe} 0}\right.$ $=6.24 \times 10^{18}$ and $1.03 \times 10^{21}$ atoms/s, respectively). The plasma density at the core-SOL boundary $n_{\mathrm{e}}^{\mathrm{LCFS}}$ is set to be $1 \times 10^{19} \mathrm{~m}^{-3}$. It should be noted that the profile of the radiation power by the carbon ions in the low iron emission rate is quite different from that in the high iron emission rate (Figs. 9 (b) and (e)) in spite of the equivalent radiation power by carbon ions $\left(P_{\mathrm{Rad}}^{\mathrm{C}}\right)$ in both cases (Fig. $\left.8(\mathrm{a})\right)$. For the low iron emission, the radiation power in the outer region of the ergodic layer is higher than that near the coreSOL boundary as shown in Fig.9(b). For the high iron emission, the radiation power near the core-SOL boundary is higher than that in the outer region (Fig. 9(e)). This difference is explained by the plasma cooling rate of the carbon ions which strongly depends on the electron tem- perature. It is found that the cooling rate of carbon ions reaches the maximum value at an electron temperature of around less than $100 \mathrm{eV}$ [13]. The region with the highest radiation power of carbon ions $\left(P_{\mathrm{Rad}}^{\mathrm{C}}\right)$ in both the low and high iron emission rates (Figs. 9 (b) and (e), respectively) corresponds to the position where the electron temperature is in this temperature range $(<100 \mathrm{eV})$ shown as light blue areas in Figs. 9 (c) and (f), respectively. In the case of the low iron emission rate, the region where the electron temperature is several tenths $\mathrm{eV}$ locates near the outer edge of the ergodic layer and the divertor legs. On the other hand, for the high iron emission rate, this region is distributed around the core-SOL boundary.

\subsection{Simulation results for the tungsten diver- tor}

Figure 10 shows the dependence of the above six parameters on the iron emission rate for the tungsten divertor. Both the radiation power by tungsten ions and the total tungsten ion content (Figs. 10 (a) and (b)) rise in the case where the iron emission rate is less than about $1 \times 10^{20}$ atoms/s. When the iron emission rate is higher than this value, these two parameters $\left(P_{\mathrm{Rad}}^{\mathrm{W}}\right.$ and $\left.Q_{\mathrm{W}+}\right)$ tend to saturate and decrease. This trend is qualitatively explained by 
Carbon Divertor, PLCFS $=12 \mathrm{MW}, \mathrm{n}_{\mathrm{e}}{ }^{\mathrm{LCFS}}=1 \times 10^{19} \mathrm{~m}^{-3}, \underline{\mathrm{IFe} 0}=6.24 \times 10^{18} \mathrm{atoms} / \mathrm{s}$
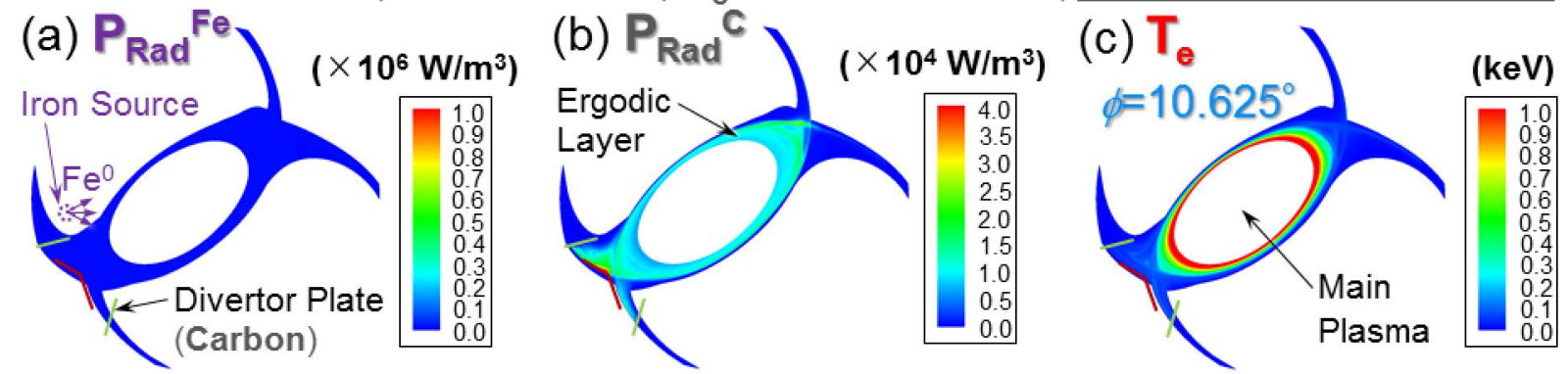

Carbon Divertor, PLCFS $=12 \mathrm{MW}, \mathrm{n}_{\mathrm{e}}{ }^{\mathrm{LCFS}}=1 \times 10^{19} \mathrm{~m}^{-3}, \underline{\mathrm{Fe} 0}=1.03 \times 10^{21}$ atoms $/ \mathrm{s}$
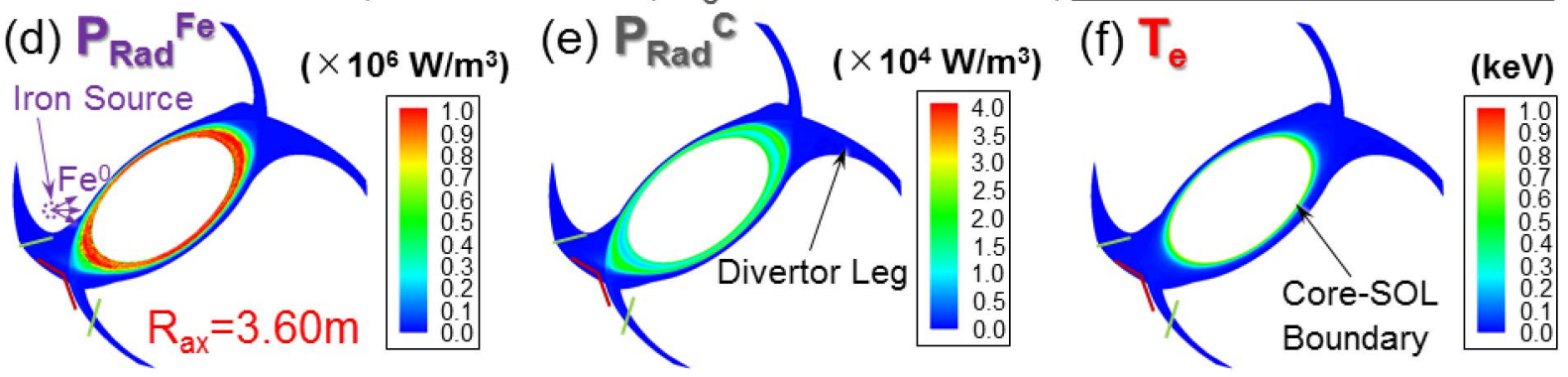

Fig. 9 The poloidal cross-section of the profile of the radiation power by the iron ions (a), by the carbon ions (b), and the electron temperature (c) for a low iron emission rate in the carbon divertor configuration. The profile of the radiation power by the iron ions (d), by the carbon ions (e), and the electron temperature (f) for a high iron emission rate is also shown.
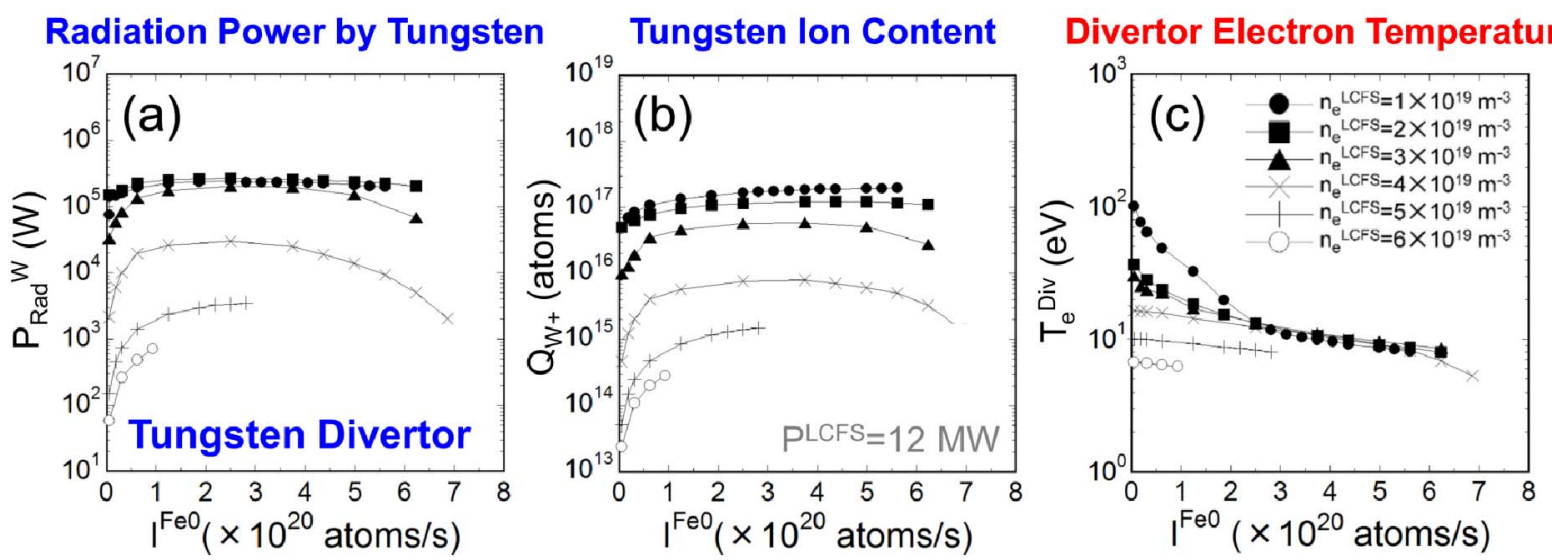

Tungsten Ion Production Rate
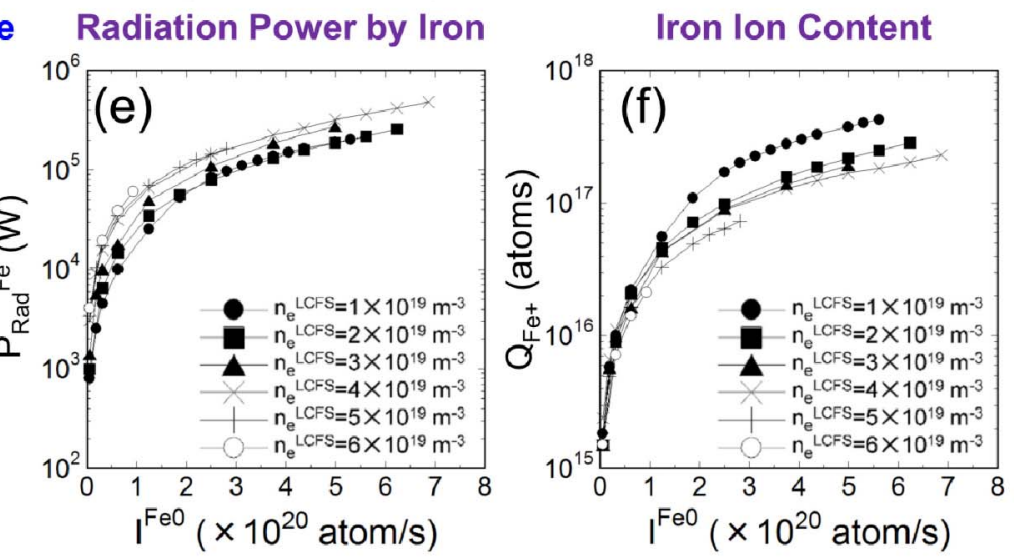

Fig. 10 The dependence of the radiation power by the tungsten ions (a), the total tungsten ion content (b), the divertor electron temperature (c), the production rate of the singly charged tungsten ions (d), the radiation power by the iron ions (e), and the total iron ion content (f) in the peripheral plasma on the iron emission rate $I^{\mathrm{Fe} 0}$ and the plasma heating power $P^{\mathrm{LCFS}}$ for the tungsten divertor in the case of $P^{\mathrm{LCFS}}=12 \mathrm{MW}$. 
Tungsten Divertor, PLCFS $=12 \mathrm{MW}, \mathrm{n}_{\mathrm{e}}^{\mathrm{LCFS}}=1 \times 10^{19} \mathrm{~m}^{-3}, \underline{\mathrm{Fe} 0}=6.24 \times 10^{18}$ atoms $/ \mathrm{s}$
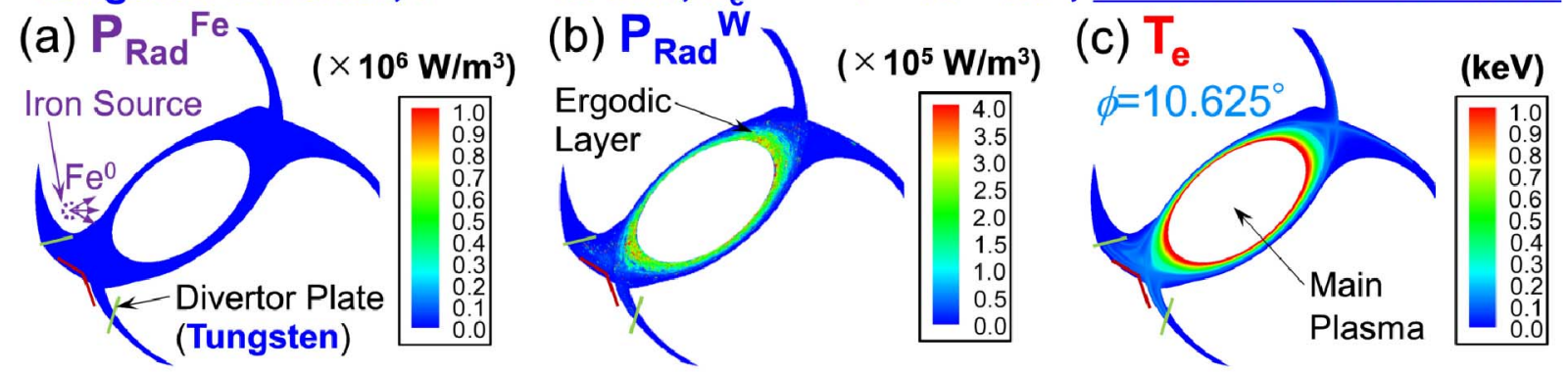

Tungsten Divertor, $\mathrm{PLCFS}^{\mathrm{L}}=12 \mathrm{MW}, \mathrm{n}_{\mathrm{e}} \mathrm{LCFS}=1 \times 10^{19} \mathrm{~m}^{-3}, \underline{\mathrm{FFe} 0}=4.37 \times 10^{20}$ atoms $/ \mathrm{s}$
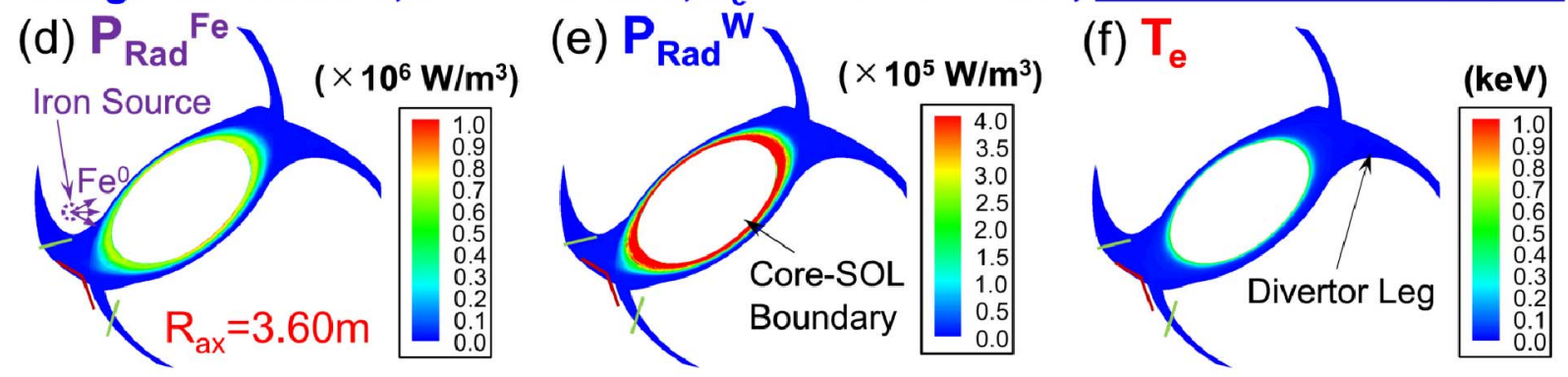

Fig. 11 The poloidal cross-section of the profile of the radiation power by the iron ions (a), by the tungsten ions (b), and the electron temperature (c) for a low iron emission rate in the tungsten divertor configuration. The profile of the radiation power by the iron ions (d), by the tungsten ions (e), and the electron temperature (f) for a high iron emission rate is also depicted.

the dependence of the production rate of the singly charged tungsten ions $\left(S_{\mathrm{W} 1+}\right)$ on the iron emission rate as shown in Fig. $10(\mathrm{~d})$. The divertor electron temperature drops with the increase in the iron emission rate $I^{\mathrm{Fe} 0}$, which is prominent in lower plasma densities $\left(n_{\mathrm{e}}^{\mathrm{LCFS}} \leq 3 \times 10^{19} \mathrm{~m}^{-3}\right)$. In this simulation, no converged solutions are obtained when the iron emission rate is more than $6-7 \times 10^{20}$ atoms/s because of the excessively low divertor electron temperature (Fig. $10(\mathrm{c})$ ). The temperature drop is caused by the radiation cooling by the iron ions, leading to the saturation of the tungsten ion production rate due to the lowered physical/self-sputtering processes (Fig. 10 (d)). This simulation proves that a catastrophic increase in the radiation power by tungsten ions due to the physical/selfsputtering of tungsten does not occur even for excessively higher iron emission rates. This is because the sputtering rate of tungsten is reduced in the lowered divertor electron temperature (Fig. 10 (c)) for the high iron emission rates. For example, the radiation power by tungsten ions $\left(P_{\mathrm{Rad}}^{\mathrm{W}}\right)$ in the low plasma density $\left(n_{\mathrm{e}}^{\mathrm{LCFS}}=1 \times 10^{19} \mathrm{~m}^{-3}\right)$ reaches the maximum value at an iron emission rate of $2.5 \times 10^{20}$ atoms/s, in which the radiation power $\left(P_{\mathrm{Rad}}^{\mathrm{W}}\right)$ is larger than the power $\left(P_{\mathrm{Rad}}^{\mathrm{C}}\right)$ for the carbon divertor by about one order of magnitude in the lower plasma density (Fig. 8 (a) and Fig. 10 (a)). On the other hand, the maximum radiation power by tungsten ions in a medium plasma density $\left(n_{\mathrm{e}}^{\mathrm{LCFS}}\right.$ $=4 \times 10^{19} \mathrm{~m}^{-3}$ ) decreases to less than that for the carbon divertor by a factor of about 2 . This simulation indicates that, for the tungsten divertor, higher plasma density operations $\left(n_{\mathrm{e}}^{\mathrm{LCFS}} \geq 4 \times 10^{19} \mathrm{~m}^{-3}\right)$ are favorable for controlling the maximum radiation power by tungsten ions $\left(P_{\mathrm{Rad}}^{\mathrm{W}}\right)$ in the case of excessively high iron emission rates.

Figure 11 presents the poloidal cross-section of the profile of the radiation power by the total iron ions, by the total tungsten ions, and the electron temperature profile in the tungsten divertor for low and high iron emission rates $\left(I^{\mathrm{Fe} 0}=6.24 \times 10^{18}\right.$ and $5.62 \times 10^{20}$ atoms/s, respectively $)$. The plasma density at the core-SOL boundary $n_{\mathrm{e}}^{\mathrm{LCFS}}$ is set to be $1 \times 10^{19} \mathrm{~m}^{-3}$. The simulation shows that the radiation power by the iron ions and the tungsten ions near the core-SOL boundary increases with the iron emission rate. The profile of the radiation power by the tungsten ions does not qualitatively change with the iron emission rate (Figs. 11 (b) and (e)), which is different from that by the carbon ions for the carbon divertor configuration. The electron temperature in the peripheral plasma significantly drops with the iron emission rate as indicated in Figs. 11 (c) and (f). That is, while the electron temperature near the core-SOL boundary in the low iron emission rate is about $1.0 \mathrm{keV}$, the temperature decreases to about $0.3 \mathrm{keV}$ in the high iron emission rate. The main cause of this temperature drop is the enhanced radiation power by both the iron and the tungsten ions in the ergodic layer around the coreSOL boundary for the high iron emission rate as shown in Figs. 11 (d) and (e).

\section{Summary}

In order to understand the characteristics of the tungsten divertor configuration in LHD plasma discharges, the comparative analysis of the impurity transport in the pe- 
ripheral plasma was performed for both the carbon and the tungsten divertor configurations using the EMC3-EIRENE in the iron dust emission case. The simulation reveals the observable difference of the dependence of the impurity transport on the plasma density in the two divertor configurations. For the carbon divertor, the radiation power, the carbon ion content, and the carbon ion production rate increases with the plasma density. On the other hand, for the tungsten divertor, the radiation power, the tungsten ion content, and the tungsten ion production rate significantly decrease with the plasma density, which indicates that higher plasma density operations are advantageous for suppressing the influence by impurity ions for the tungsten divertor. The investigation of the dependence of the impurity transport on the iron emission rates shows the quite different trend in both the two divertor configurations. For the carbon divertor, the radiation power by carbon ions does not significantly change with the iron emission rate. For the tungsten divertor, while the radiation power by tungsten ions rises in the lower iron emission rates, the radiation power tends to saturate and decrease in the higher iron emission rates. The simulation proves that a catastrophic increase in the radiation power by sputtered tungsten in excessively higher iron dust emission cases does not occur due to the lowered divertor electron temperature, which is a favorable characteristic in the tungsten divertor configuration to sustain long pulse discharges in LHD. The simulation also reveals that, for the tungsten divertor, higher plasma density operations $\left(n_{\mathrm{e}}^{\mathrm{LCFS}} \geq 4 \times 10^{19} \mathrm{~m}^{-3}\right.$ ) are effective for controlling the maximum radiation power by tungsten ions even in the case of excessively higher iron emission rates, in which the radiation power $\left(P_{\mathrm{Rad}}^{\mathrm{W}}\right)$ is suppressed to less than that $\left(P_{\mathrm{Rad}}^{\mathrm{C}}\right)$ for the conventional carbon divertor by a factor of about 2 .

\section{Acknowledgments}

This work is performed under the auspices of the NIFS Collaboration Research program (NIFS18KNXN359). One of the authors (M.S.) would like to thank Y. Feng for permission to use the EMC3-EIRENE. He is also grateful for the computational resources of the LHD numerical analysis server and the plasma simulator in NIFS. This work is supported by JSPS KAKENHI Grant Number $16 \mathrm{H} 04619$.

[1] M. Shoji et al., Nucl. Fusion 55, 053014 (2015).

[2] M. Tokitani et al., J. Nucl. Mater. 463, 91 (2015).

[3] M. Shoji et al., Plasma Fusion Res. 11, 2402056 (2016).

[4] Y. Feng et al., Plasma Phys. Control. Fusion 44, 611 (2002).

[5] G. Kawamura et al., Contrib. Plasma Phys. 54, 437 (2014).

[6] H. Summers et al., 2004 The ADAS User Manual (version 2.6), http://adas.phys.strath.ac.uk

[7] W. Eckstein et al., Computer Simulation of Ion Solid Interaction (Springer, Berlin, 1991) p.169.

[8] V. B. Mech et al., J. Nucl. Mater. 255, 153 (1998).

[9] M. Shoji et al., Nucl. Mater. Energy 17, 188 (2018).

[10] A. Kirschner et al., Contrib. Plasma Phys. 56, 622 (2016).

[11] M. Kobayashi et al., Contrib. Plasma Phys. 48, 255 (2008).

[12] Y. Takeiri et al., Nucl. Fusion 57, 102023 (2017).

[13] J. Wesson, Tokamaks (Clarendon Press, Oxford, 1997) p.209. 